\title{
Analysis of scour characteristics in presence of aerated crossing jets
}

STEFANO PAGLIARA, Prof., Dept. of Civil Engineering, University of Pisa, Via Gabba 22, 56122 Pisa, Italy, phone +39050 2217717; Fax: +39 050 2217730; email:s.pagliara@ing.unipi.it

MICHELE PALERMO, Ph.D., Dept. of Civil Engineering, University of Pisa, Via Gabba 22, 56122 Pisa, Italy; e-mail:michele.palermo@ing.unipi.it

\begin{abstract}
Jets scour is a major topic in hydraulic engineering. It has to be carefully analyzed in order to understand the mechanism and predict its geometry. The jets configuration has a deep influence on the scour features. In the present paper the analysis was conducted in presence of two symmetric crossing jets, varying the discharge, the air content, the tailwater level in the downstream stilling basin, the angle between the jets and the vertical distance of the jets crossing point from the water surface, for different vertical jets angle. It was proven that the presence of the air in the jets deeply affects the scour morphology. The scour geometry was analyzed and compared with the respective obtained in black water conditions. Useful practical relationships are proposed to estimate the main scour hole dimensions. The analysis was also extended to non dimensional profiles and it was proved that the effect of air content on them is negligible.
\end{abstract}

Keywords: Air content, Crossing jets, Dam, Plunge pool, Scour

\section{INTRODUCTION}

The presence of air in the plunging jets is an occurrence that happens in real structures and has to be taken seriously in consideration in order to understand the scour mechanism and avoid structural risks. The scour hole dimensions are influenced by the quantity of air which is entrained by plunging jets downstream of dams. In particular, the effect of air entrainment on scour features becomes much more prominent in presence of crossing jets, such as in the case of arch dams. Generally, the outlets of arch dams are located symmetrically and the jets cross before entering in the downstream water body (see for example the Hoover Dam, USA). Thus, a huge quantity of air entrainment occurs, due to both jet enlargement during the free fall and the jets spread beyond the crossing point. The air quantity in the resulting jet beyond the crossing point is very difficult to quantify, but the effect of air presence in the plunging 
jets, before they cross each other, can be experimentally evaluated in terms of scour hole morphology variations. This type of configuration can be of interest for practical applications, as generally it can lead to a reduction of the erosive capacity of the resulting jet beyond the crossing point, due to the huge energy dissipation and a relevant splash occurring.

In the technical literature many studies are present dealing with scour phenomenon due to plunging jets, in the absence of air entrainment (i.e., black water conditions). Mostly, the previous studies are relative to single plunging jets and countermeasures that can be adopted in order to reduce scour lengths or prevent structural collapse. In particular, the effect of bed material on scour morphology due to plunging jets was taken into consideration by Rajaratnam and Berry (1977) who investigated the scour phenomenon in the presence of both sand and polystyrene beds. Several authors conducted experiments in black water conditions in order to understand the most important variables on which the erosive process depends, both in presence and in absence of protection structures (among these, Rajaratnam and Macdougall (1983), Mih (1982), Mih and Kabir (1983), Pagliara et al. (2008), Pagliara and Palermo (2008) and Pagliara et al. (2010)). In particular, it was experimentally proven that, in the case of a single plunging jet and in the absence of any protection structure, the scour hole dimensions mainly depend on the jet discharge, water level in the downstream water body (tailwater), jet inclination and the submergence condition of the jet (i.e. if the jet outlet is submerged or not). Generally, all the previous studies furnish experimental relationships to evaluate the scour features. Based on application of Newton's second law, an attempt to furnish a more theoretical approach to foresee the scour depth was developed by Hoffmans (1998). However, relatively few studies take into consideration the air presence in plunging jets and its effect on scour hole morphology (among these, Pagliara et al. (2006), and Pagliara et al. (2009)). Ervine and Falvey (1997) studied the behavior of water jets plunging through the atmosphere, analyzing the physical properties of the jets and their spreading. Furthermore, Manso et al. (2007) conducted a comprehensive analysis on the dynamic pressures at the issuance and at the bottom of the pool investigating velocities and aeration conditions similar to those occurring in prototypes.

Even less studies analyze the scour process due to crossing jets. In addition, they are relative to peculiar cases such as in Li et al.(2006). Recently, a study on crossing jets in the absence of air was proposed by Pagliara et al. (2011a) for black water conditions. They analyzed the influence of several parameters (i.e. angle between crossing jets, tailwater level, distance between the crossing point and the downstream water level varying the vertical angle). 
According to authors' knowledge, no studies were conducted till date which investigate the effect of air on scour features in presence of crossing jets. Thus, the aim of the present paper is to analyze the scour morphology in presence of two symmetric crossing jets, varying the discharge, the air content, the tailwater level in the downstream stilling basin, the crossing angle between the jets and the vertical distance of the jets crossing point from the water surface, for different vertical jets angles. It was proven that the presence of the air in the jets affects the scour morphology. The scour depths were analyzed and some useful empirical relationships were derived to predict the scour hole geometry. In addition, the analysis was extended to non dimensional profiles and it was proved that the air presence does not modify them significantly.

\section{EXPERIMENTAL SETUP}

Experiments were conducted in a channel $6 \mathrm{~m}$ long, $0.8 \mathrm{~m}$ wide and $0.7 \mathrm{~m}$ high. One material was used whose granulometric characteristics are $d_{10}=7.17 \mathrm{~mm}, d_{16}=7.49 \mathrm{~mm}, d_{84}=10.02 \mathrm{~mm}$, $d_{90}=10.26 \mathrm{~mm}$, the non-uniformity parameter $\sigma=\left(d_{84} / d_{16}\right)^{1 / 2}=1.16$ and the density $\rho_{s}=2453$ $\mathrm{kg} / \mathrm{m}^{3}$. The crossing jets were simulated using two pipes of internal diameter $D=0.022 \mathrm{~m}$. The air was supplied in the pipes using an air compressor. Air discharge was regulated using two valves (valve A and B) and measured by an air flowmeter installed in the air circuit. Figure 1c show a picture of the experimental apparatus. The experiments were conducted varying both air discharge $Q_{A}$ and water discharge $Q_{W}$, namely $01 / \mathrm{s}<Q_{A}<1.251 / \mathrm{s}$ and $1.251 / \mathrm{s}<Q_{W}<4.21 / \mathrm{s}$. It is worth noting that the total water discharge and air discharge were subdivided equally in both the symmetric crossing pipes, meaning that in each pipe the water discharge is $Q_{W} / 2$ and the air discharge is $Q_{A} / 2$. In particular, reference tests were conducted in the same hydraulic and geometric configuration but in absence of air $\left(Q_{A}=0\right)$. The total discharge $Q_{A W}$ is equal to $Q_{A}+Q_{W}$. It has to be noted that varying both $Q_{A}$ and $Q_{W}$ causes a variation of air-water mixture densimetric Froude number $F_{A W}$. This non dimensional parameter was introduced by Canepa and Hager (2003). It is defined as $F_{A W}=V_{A W} /\left(g^{\prime} d_{90}\right)^{1 / 2}$ with $V_{A W}=\left(Q_{W}+Q_{A}\right) /\left(\pi D_{e q}{ }^{2} / 4\right), g^{\prime}=\left[\left(\rho_{s^{-}}\right.\right.$ $\rho) / \rho] g$ as the reduced gravitational acceleration with the densities $\rho_{s}$ and $\rho$ of sediment and water, respectively. Note that in the case of one single pipe (as in the experiments conducted by Canepa and Hager, 2003) $D_{e q}$ is the internal diameter of the pipe. Whereas, in the case of two crossing pipes $D_{e q}$ is the equivalent diameter, i.e. the diameter of the single pipe having the same total area of the two crossing pipes. It implies that $D_{e q}=\left(2 D^{2}\right)^{0.5}=0.0311 \mathrm{~m}$ for the present experiments. It can be easily noted that $V_{A W}=\left(Q_{W}+Q_{A}\right) /\left(\pi D_{e q}{ }^{2} / 4\right)=V_{W}(1+\beta)$, where $V_{W}$ $=\left(Q_{W}\right) /\left(\pi D_{e q}{ }^{2} / 4\right)$ and $\beta=Q_{A} / Q_{W}$ as the dimensionless flow rate. It implies that $F_{A W}=F_{W}(1+\beta)$, 
where $F_{W}=V_{W} /\left(g^{\prime} d_{90}\right)^{1 / 2}$, which is the densimetric Froude number of the black water jet. It has to be noted that in the case in which no air is supplied in the pipe, $\beta=0$, thus $F_{A W}=F_{W}$. In the present tests $8.61<F_{A W}<14.47$ and $0<\beta<1$. The experiments were carried out for different downstream water levels $h_{0}$ and the non dimensional tailwater level $T_{w}=h_{0} / D_{e q}$ ranged between 0.7 and 7.1. Moreover, different crossing jets angles $\alpha_{c}$ (i.e. the crossing angle between the jets) were tested, i.e., $\alpha_{c}=30^{\circ}, 75^{\circ}$ and $120^{\circ}$, respectively. The vertical angle of the crossing pipes plane respect to the horizontal plane $\alpha_{v}$ was also varied. In particular, three $\alpha_{v}$ were tested: $60^{\circ}, 75^{\circ}$ and $85^{\circ}$. Another important tested parameter was the vertical distance between the crossing point of the jets and the original bed level $S$, and in non dimensional form it can be written as $\delta=S / D_{e q}$. Experiments were conducted for $0<\delta<6.5$. Figure $1 \mathrm{a}-\mathrm{b}$ shows a sketch of the experimental apparatus in which all the main geometric parameters cited above are illustrated. Moreover, in the same Fig. 1a-b, also the characteristic dimensions of the scour hole are reported. In particular, $z_{m c A}$ is the maximum scour hole depth, $l_{c A}$ is the scour hole length, $z_{M c A}$ is the dune height, $b_{c A}$ is the maximum scour width (section E-E), $l_{M c A}$ is the dune length (in the axial section $\mathrm{A}-\mathrm{A}$ ), $b_{M c A}$ is the maximum dune width (in the section C-C where $z=z_{M c A}$ ) and $B$ is the channel width. $x, y$, and $z$ are the longitudinal transversal and vertical coordinates of the reference system, respectively, and 0 is the origin of the reference system. The subscript A used in the symbols is referred to the case in which air is present in the jets, whereas when $\beta=0$ (i.e. reference tests) the symbols indicating the lengths are the same without the subscript A (for example $z_{m c}$ is the maximum scour depth in the absence of air in the jets). About 200 tests were conducted. The characteristic dimensions of the scour hole were measured in dynamic conditions (i.e. when the jets are on and the scour geometry reached its dynamic equilibrium configuration). Generally the dynamic equilibrium configuration was reached after 40 minutes. Measurements of both water levels and scour morphology were taken with a special point gauge fitted with a circular plate at the bottom as in Canepa and Hager (2003). 

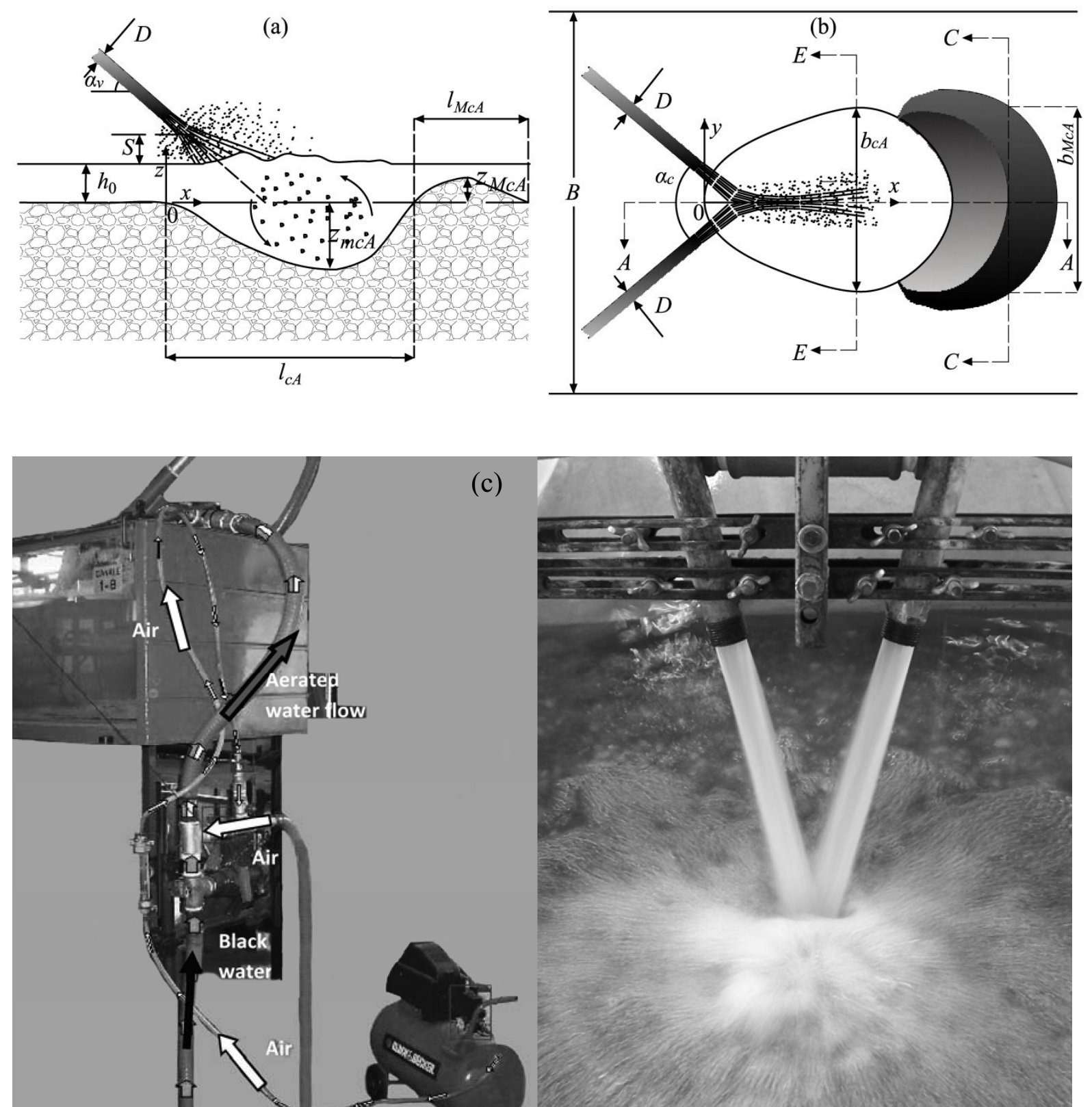

(c)



Figure 1 Diagram sketch and picture of the experimental apparatus with the indication of the geometric and hydraulic parameters

\section{RESULTS AND DISCUSSION}

\subsection{Scour morphology classification}

The scour process depends on several parameters which can strongly modify the scour hole geometry. The combination of all the geometric and hydraulic variables caused different scour hole and dune morphologies. Namely, according to Pagliara et al (2011b), three different scour hole types take place in the tested range of parameters.

The Type 1 is characterized by an approximately circular shape of the scour hole and a dune 
which is not extended in length but it can either partially or totally surround the scour hole. Type 2 presents a quasi-elliptical shape of the scour hole and a dune which is located completely in the downstream side of the scour hole and it is characterized by a milder surface slope than that of Type 1 . Type 3 is characterized by a longitudinally extended downstream dune, whose length is much larger than the scour hole longitudinal extension and presents a very mild slope. Finally, Type 4 represents the configuration in which no scour takes place, i.e. when the erosive capacity of the jet is not enough to move the bed material. In Figure 2 a-c pictures relative to the first three types are reported (see Pagliara et al. 2011b).

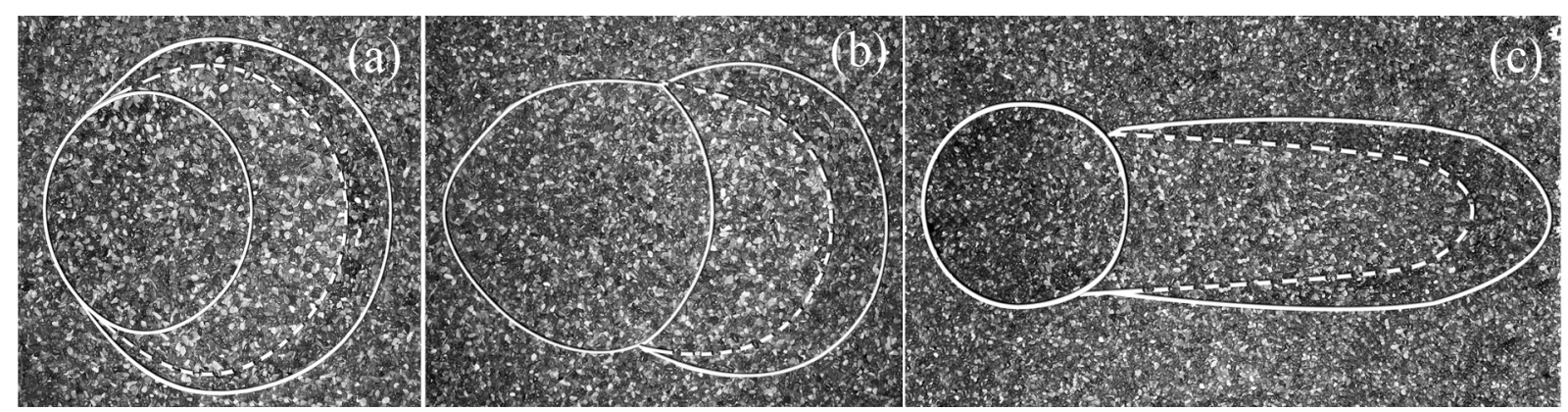

Figure 2 Pictures of the three different scour types: (a) Type 1, (b) Type 2 and (c) Type 3 (flow from the left)

The four scour types occur for different hydraulic and geometric conditions. For $\beta>0.25$ (minimum tested case in the presence of air), Figure 3a-c (Pagliara et al. 2011b) illustrates the existence fields of the distinguished scour types. A morphological analysis allowed to assess that the different scour types existence fields mainly depend on crossing angles between the jets $\alpha_{c}$, tailwater level $T_{w}$ and the non dimensional vertical distance $\delta$ between the jets crossing point and the water surface. Thus, three graphs $T_{w}(\delta)$, valid for different crossing angles $\alpha_{c}$, were plotted. The existence fields were delimitated and both the effect of $\alpha_{v}$ and $\beta$ (bold arrows) on the boundary regions (hatched lines) are reported. 
(a) $\alpha_{c}=30^{\circ}$

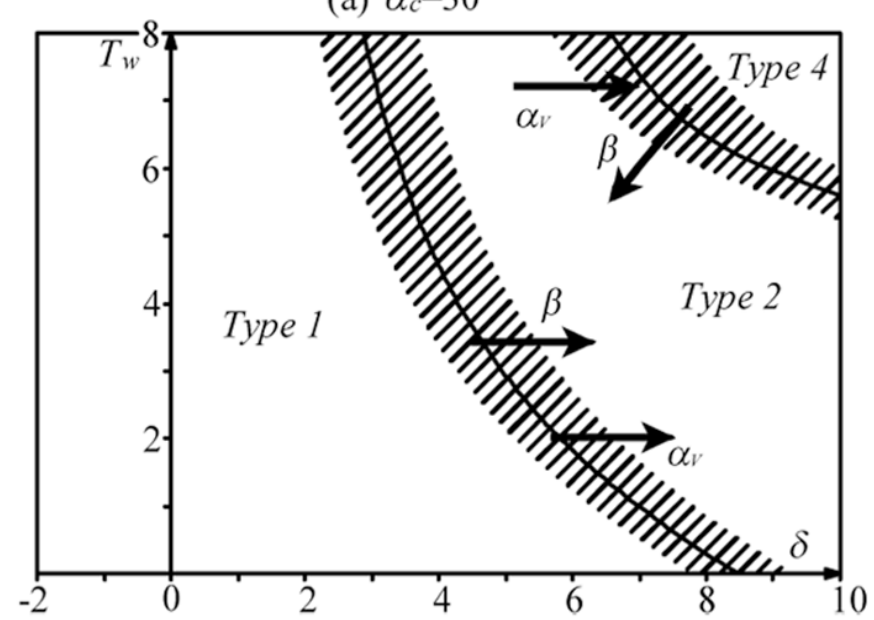

(b) $\alpha_{c}=75^{\circ}$

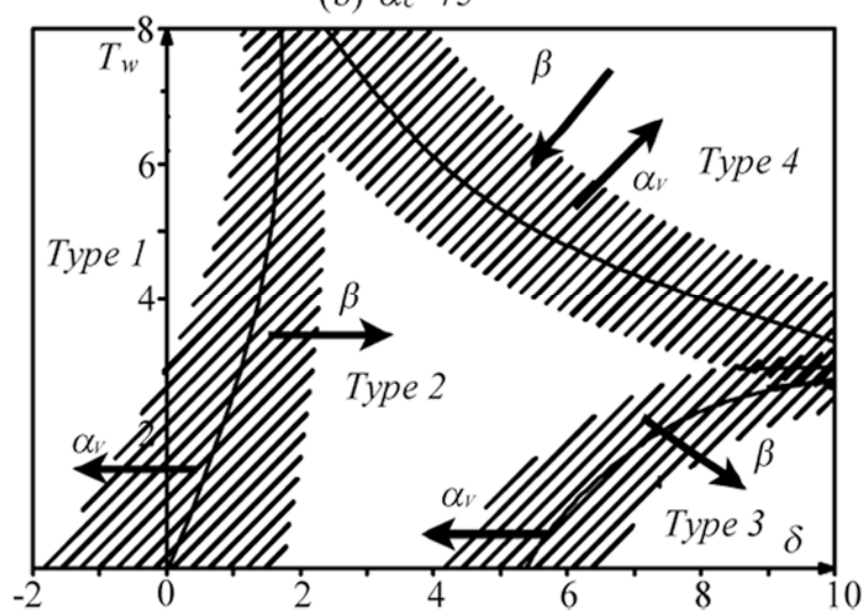

(c) $\alpha_{c}=120^{\circ}$

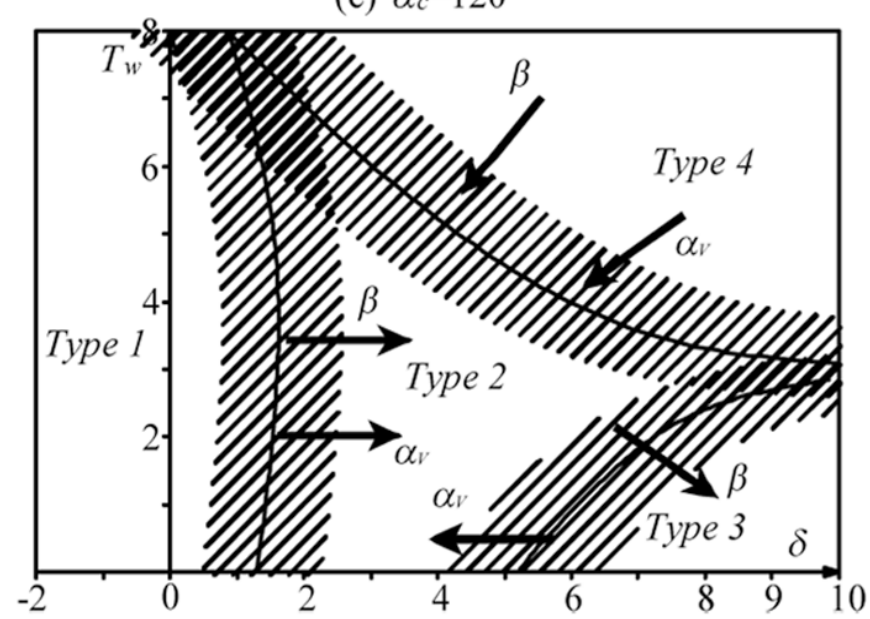

Figure 3 Scour Types existence fields for $\beta>0.25$ and all tested vertical angles: (a) $\alpha_{c}=30^{\circ}$, (b) $\alpha_{c}=75^{\circ}$, and (c) $\alpha_{c}=120^{\circ}$

Figure 3a shows that only three scour types (i.e., Type 1, 2 and 4) take place for $\alpha_{c}=30^{\circ}$. The transition between Type 1 and 2 occur for $\delta>4$, but higher values of $\delta$ are required when both $\alpha_{v}$ and $\beta$ increases. This behavior is not confirmed for the transition between Type 2 and 4 , as 
shown in Figure 3a. A similar morphological analysis was conducted also for $\alpha_{c}=75^{\circ}$ and $\alpha_{c}=120^{\circ}$. In the first case $\left(\alpha_{c}=75^{\circ}\right.$, see Figure 3b), Type 1 mainly occurs for $T_{w}>3$ and $\delta<2$, Type 2 for $T_{w}<6$ and $2<\delta<6$, Type 3 for $T_{w}<3$ and $\delta>5$, and Type 4 generally for $T_{w}>4$ and $\delta>2$. Similar existence fields for various scour types can be observed for $\alpha_{c}=120^{\circ}$ (see Figure 3c). Also for $\alpha_{c}=75^{\circ}$ and $120^{\circ}$, the effect of both the parameters $\beta$ and $\alpha_{v}$ on the transition region was pointed out and reported in Figure 3b-c, respectively.

\subsection{Effect of air presence on scour hole depth}

The presence of air in the jets affects the scour dimensions. In particular, the scour hole depth is the most relevant parameter which has to be analyzed. A comparison of the maximum non dimensional scour depth both in presence and absence of air was conducted for all the tests, in the same geometric and hydraulic conditions (i.e., same $\delta, T_{w}, \alpha_{c}$, and $\alpha_{v}$ ), and for the same $F_{A W}$. Thus, the scour depth occurring in black water conditions for a defined $F_{A W}=F_{W}$ were compared with those occurring for the same $F_{A W}$, but varying the dimensionless flow rate $\beta$. The results were plotted in graphs $Z_{m c A} / Z_{m c}$ versus $\beta$, in which $Z_{m c A}=z_{m c A} / D_{e q}$ and $Z_{m c}=z_{m c} / D_{e q}$ are the non dimensional scour hole depths in the presence and absence of air in the jets, respectively. A preliminary analysis allowed to establish that increasing $\beta$, and keeping $F_{A W}$ constant, the scour depth always reduces. The reduction is also dependent on $T_{w}$ and $\delta$, for each $\alpha_{c}$, and $\alpha_{v}$ configurations tested. But a clear and unique trend cannot be deducted varying both $T_{w}$ and $\delta$. In fact, the combination of these two last parameters has a different effect on scour reduction for various angle configurations tested. However, it was experimentally proved that the average reduction is almost the same for both $\alpha_{c}=30^{\circ}$ and $\alpha_{c}=75^{\circ}$ and all $\alpha_{v}$ tested, whereas for $\alpha_{c}=120^{\circ}$ it results to be more prominent. In Figure $4 \mathrm{a}-\mathrm{b}$ two graphs are reported by which it is possible to understand the average reduction effect of $\beta$ on scour hole depths.

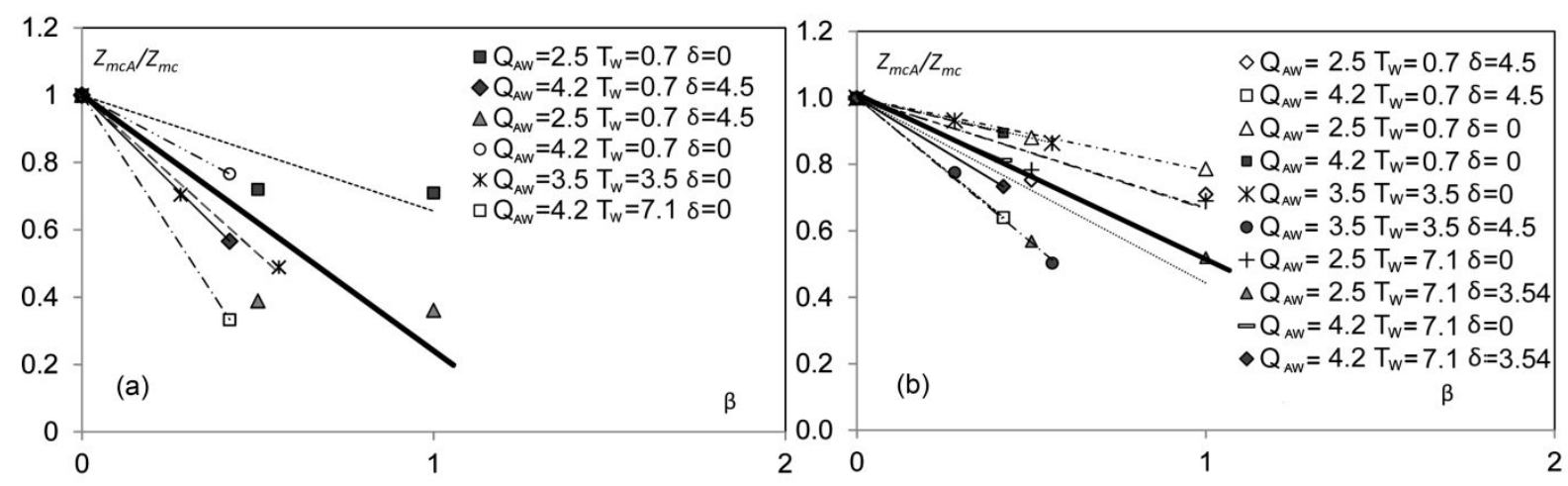


Figure $4 Z_{m c A} / Z_{m c}$ versus $\beta$, for various $\delta, T_{w}$ and $Q_{A W}$ tested and for (a) $\alpha_{v}=60^{\circ}$ and $\alpha_{c}=120^{\circ}$ and (b)

$$
\alpha_{v}=75^{\circ} \text { and } \alpha_{c}=30^{\circ}
$$

In Figure $4 \mathrm{a}$, a graph $Z_{m c} / Z_{m c}$ versus $\beta$ is reported and it is valid for $\alpha_{v}=60^{\circ}$ and $\alpha_{c}=120^{\circ}$. The bold line represents the average reduction that occurs for $\alpha_{c}=120^{\circ}$. It can be observed that increasing $\beta$ the average reduction is very prominent. Similarly, in Figure $4 \mathrm{~b}$ a graph $Z_{m c} / Z_{m c}$ versus $\beta$ is reported and it is valid for $\alpha_{v}=75^{\circ}$ and $\alpha_{c}=30^{\circ}$. As specified above, a similar average behaviour is also valid for $\alpha_{c}=75^{\circ}$. In these last two cases, the reduction due to increasing $\beta$ is less prominent than for $\alpha_{c}=120^{\circ}$.

\subsection{Evaluation of scour hole dimensions}

For design purposes, it is very important to predict the geometric dimensions of the scour hole. Pagliara et al. (2011a) proved that, for black water conditions, the non dimensional scour hole lengths depend on the combined effects of five independent parameters: $F_{W}$ (which is equal to $F_{A W}$ in black water conditions), $T_{w}, \delta, \alpha_{v}$ and $\alpha_{c}$. Due to the complexity of the phenomenon, it is not possible to point out individual effects of each governing parameters on the scour hole features. Thus, Pagliara et al. (2011a) conducted a separate analysis for each $\alpha_{c}$ tested, as the crossing angle between the pipes was identified as the most significant designindependent parameter. This is mainly due to the fact that varying $\alpha_{c}$ the scour process significantly changes. In fact, the transversal velocity components of the jets increases with $\alpha_{c}$, resulting in an increased jet splash. Consequently, the scour hole morphology is extremely influenced by this last parameter, as shown in Figure 3. Based on these considerations, Pagliara et al. (2011a) proposed the following empirical relationship to predict various scour length parameters

$\varphi=a_{1}+a_{2} \cdot T_{w}+a_{3} \cdot F_{W}+a_{4} \cdot \delta+a_{5} \cdot \alpha_{v}+a_{6} \cdot T_{w} \cdot F_{W}+a_{7} \cdot T_{w} \cdot \delta+a_{8} \cdot F_{W} \cdot \delta+a_{9} \cdot T_{w} \cdot \alpha_{v}+a_{10} \cdot F_{W} \cdot \alpha_{v}+a_{11} \cdot \delta \cdot \alpha_{v}(1)$

where, $\varphi$ is the non dimensional dependent variable, i.e., $\varphi=Z_{m c}=z_{m c} / D_{e q}, \varphi=L_{c}=l_{c} / D_{e q}$ or $\varphi=B_{c}=b_{c} / D_{e q}$, according to which non dimensional scour hole length is taken into consideration. $z_{m c}$ is the dimensional scour hole depth, $l_{c}$ is the dimensional scour hole length, and $b_{c}$ is the dimensional scour hole width in black water conditions. $a_{i}$ are the coefficients of Eq. (1), in which the subscript $i$ varies between 1 and 11 and they are reported in Table 1 . In addition, Eq. (1) is valid for $\varphi \geq 0$. If the calculated value of $\varphi$ is negative, it implies that no scour process takes place, for the selected combinations of the independent parameters. Thus, in this case, $\varphi$ has to be assumed equal to 0 and scour Type 4 occurs (see Fig. 3). For 
intermediate $\alpha_{c}$ values (i.e., $30^{\circ}<\alpha_{c}<75^{\circ}$ and $75^{\circ}<\alpha_{c}<120^{\circ}$ ), Pagliara et al. (2011a) proposed that the desired dependent variable $\varphi$ can be estimated by linear interpolation of the values given by Eq. (1) in the extreme limits of the $\alpha_{c}$ intervals.

Table 1 Coefficients $a_{i}$ of Eq. (1) for $\alpha_{c}=30^{\circ}, 75^{\circ}$, and $120^{\circ}$ and for non dimensional scour hole lengths in black water conditions.

\begin{tabular}{|c|c|c|c|c|c|c|c|c|c|}
\hline \multirow{2}{*}{$a_{i}$} & \multicolumn{3}{|c|}{$Z_{m c}$} & \multicolumn{3}{|c|}{$L_{c}$} & \multicolumn{3}{|c|}{$B_{c}$} \\
\hline & $30^{\circ}$ & $75^{\circ}$ & $120^{\circ}$ & $30^{\circ}$ & $75^{\circ}$ & $120^{\circ}$ & $30^{\circ}$ & $75^{\circ}$ & $120^{\circ}$ \\
\hline$\overline{a a_{1}}$ & 4.938721 & 5.223549 & 1.717833 & 11.10140 & -0.756665 & 11.51507 & 7.049030 & 14.47283 & 12.25137 \\
\hline$a_{2}$ & -0.336533 & -0.945931 & -0.661428 & -0.47634 & -0.973429 & -2.13863 & -0.550723 & -1.82278 & -1.69188 \\
\hline$a_{3}$ & 0.157614 & 0.382553 & 0.486517 & 1.06130 & 2.434837 & 1.22969 & 1.172421 & 0.97831 & 1.56659 \\
\hline$a_{4}$ & 0.245859 & -0.506431 & -0.180330 & 0.93142 & 0.041244 & -0.38370 & 0.544552 & -1.17873 & -0.95224 \\
\hline$a_{5}$ & -0.093217 & -0.071007 & -0.017984 & -0.18468 & 0.034934 & -0.11042 & -0.115642 & -0.17800 & -0.21059 \\
\hline$a_{6}$ & -0.002492 & -0.012897 & -0.006818 & 0.01044 & -0.085776 & -0.00112 & -0.000236 & -0.05127 & -0.11303 \\
\hline$a_{7}$ & -0.099869 & -0.086593 & -0.074767 & -0.27832 & -0.455667 & -0.47252 & -0.244566 & -0.20367 & -0.42152 \\
\hline$a_{8}$ & 0.002565 & -0.009843 & -0.006365 & 0.05855 & -0.020578 & 0.00926 & 0.039614 & 0.03242 & -0.04766 \\
\hline$a 9$ & 0.008269 & 0.010977 & 0.005013 & 0.01918 & 0.019608 & 0.01710 & 0.020547 & 0.02493 & 0.03060 \\
\hline$a_{10}$ & 0.003650 & 0.000086 & -0.002834 & -0.00412 & -0.019714 & -0.00445 & -0.006422 & 0.00258 & -0.00213 \\
\hline$a_{11}$ & 0.001518 & 0.006988 & 0.001927 & -0.00331 & 0.020090 & 0.02071 & 0.006407 & 0.01016 & 0.03497 \\
\hline
\end{tabular}

For the current experiments, the effect of $\beta$ was taken into account. The experimental data were carefully analysed and Eq. (1) was modified introducing a multiplicative function $f(\beta)$. The following relationship was found:

$\varphi_{A}=\varphi \cdot f(\beta)=\varphi(\beta \cdot m+1)$

where, $\varphi$ is calculated using Eq. (1), in which $F_{W}$ is replaced by $F_{A W} \varphi_{A}$ is the dependent scour hole parameter in the presence of air in the jets, i.e., $\varphi_{A}=Z_{m c A}, \varphi_{A}=L_{c A}=l_{c A} / D_{e q}$ and $\varphi_{A}=B_{c A}=b_{c a} / D_{e q} . m$ is a coefficient depending on $\alpha_{c}$, whose values are reported in Table 2. Analyzing the values of the coefficient $m$ in Table 2, several considerations can be deduced. $\mathrm{Z}_{m c A}$ decreases with $\beta$ and $\alpha_{c}$, as also proved by Figure 4 . This is mainly due to the fact that the resulting air-water mixture jet has less erosive capacity than a black water jet. The same effect can be pointed out for $L_{c A}$. Whereas, the scour hole width $B_{c A}$ behaves differently. In fact, tends to slightly increase with $\beta$ as well as with $\alpha_{c}$. Higher crossing angles cause radial splash and higher air content in the jet amplifies the mentioned radial splashing action, resulting in a shallower and shorter but wider scour hole.

Note that for $\beta=0$, the multiplicative function $f(\beta)=(\beta \cdot m+1)=1$ and $F_{W}=F_{A W}$, resulting in $\varphi_{A}=\varphi$. It means that Eq. (2) coincides with Eq. (1) for black water conditions. Equation (2) can be applied to the generality of cases in the tested range of parameters and it has the advantage to 
take into account the jet air content. Figure 5a-c shows the comparison between measured and calculated values of $Z_{m c A}, L_{c A}$ and $B_{c A}$, using Eq. (2). It can be easily observed that the proposed general equation well predicts the totality of data, within a range of $30 \%$ deviation. The same considerations for $\beta=0$ are still valid for Eq. (2). In particular, if $\varphi_{A}<0$ it means that no scour process takes place, thus $\varphi_{A}$ has to be assumed equal to 0 . In addition, for intermediate $\alpha_{c}$ values (i.e., $30^{\circ}<\alpha_{c}<75^{\circ}$ and $75^{\circ}<\alpha_{c}<120^{\circ}$ ), $\varphi_{A}$ can be estimated by linear interpolation of the values given by Eq. (2) in the extreme limits of the $\alpha_{c}$ intervals.

Table 2 Values of $m$ in Eq. 2.

\begin{tabular}{|c|c|c|c|}
\hline \multicolumn{4}{|c|}{ Values of $m$} \\
\hline$\alpha_{c}$ & $Z_{m c A}$ & $L_{c A}$ & $B_{c A}$ \\
\hline $30^{\circ}$ & -0.350 & -0.050 & 0.094 \\
\hline $75^{\circ}$ & -0.390 & -0.164 & 0.280 \\
\hline $120^{\circ}$ & -0.590 & -0.280 & 0.300 \\
\hline
\end{tabular}

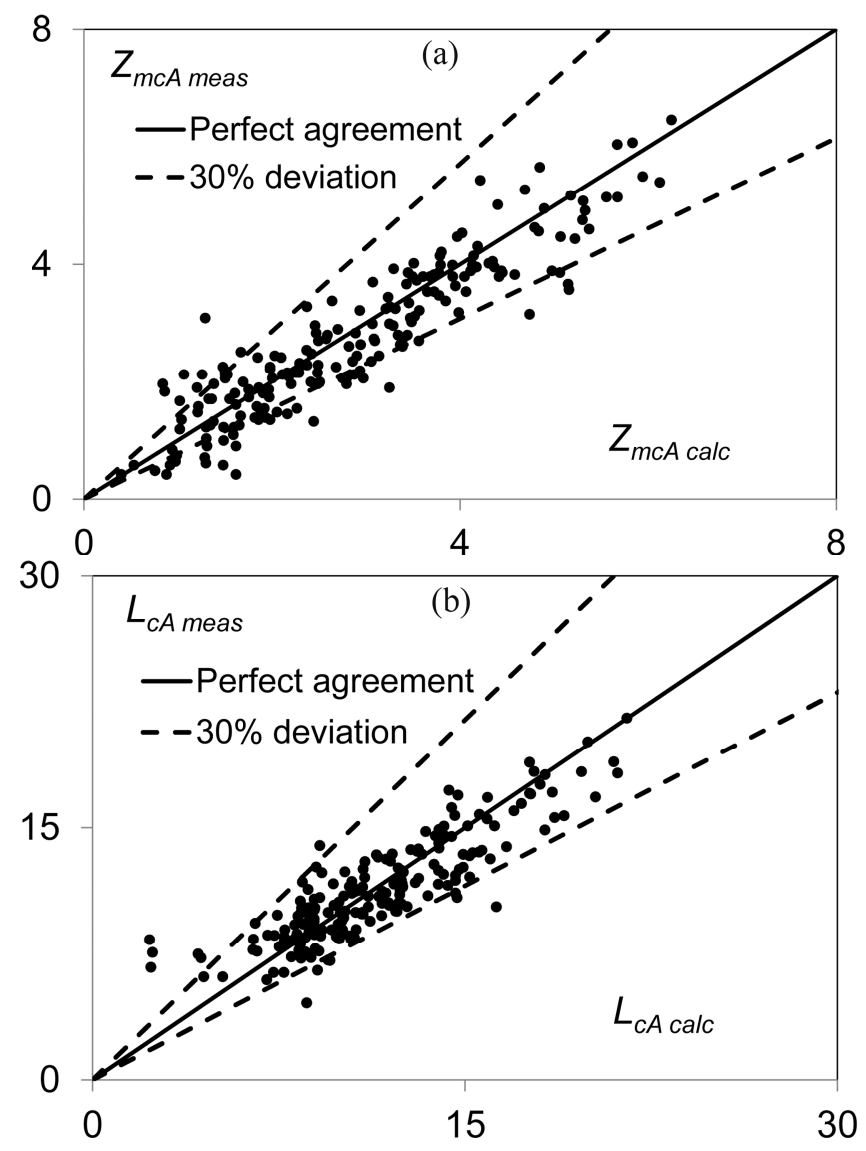




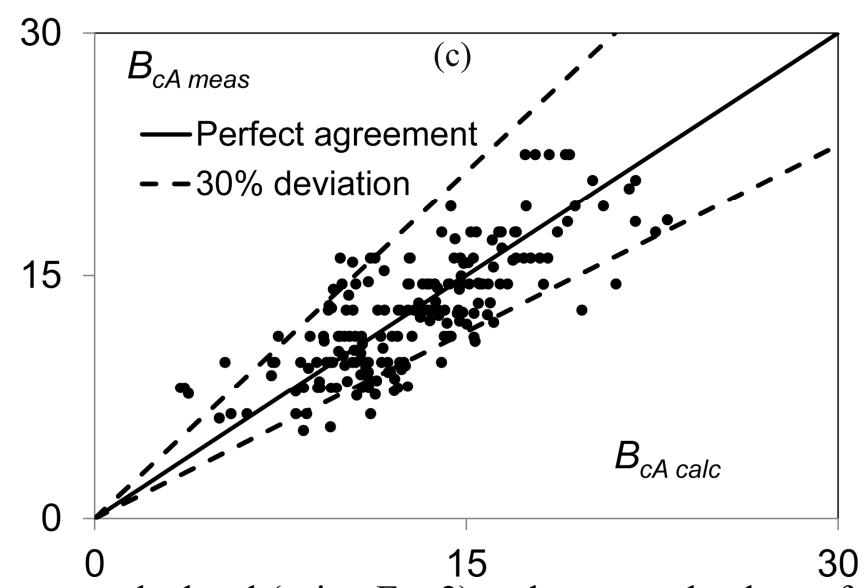

Fig. 5. Comparison between calculated (using Eq. 2) and measured values of (a) $Z_{m c A}$, (b) $L_{c A}$ and (c) $B_{c A}$.

\subsection{Scour hole and dune profiles similitude analysis}

A further analysis was conducted in order to check the effect of air presence on non dimensional profiles and to find out if any similitude among them can be established. According to the reference system adopted in Figure $1 \mathrm{a}-\mathrm{b}, X=x / l_{c A}$ is the non dimensional longitudinal coordinate (in the section A-A, see Figure $1 \mathrm{~b}$ ), $Z=z / z_{m c A}$ is the non dimensional vertical coordinate and $Y=\left(y+b_{c A} / 2\right) / b_{c A}$ (in the section E-E) is the non dimensional transversal coordinate. Both the longitudinal and transversal non dimensional profiles were plotted in graphs $Z(X)$ and $Z(Y)$, respectively, for all the tested conditions. For black water conditions $(\beta=0)$, Pagliara et al (2011a) proposed the following Eqs. (3)-(5) for the longitudinal scour hole profiles and Eq. (6) for the transversal scour hole profile.

$Z=-1.30 X^{4}+7.09 X^{3}-4.66 X^{2}-1.13 X$

Valid for $\alpha_{c}=30^{\circ}$

$Z=-3.51 X^{5}+5.46 X^{4}+0.25 X^{3}+0.59 X^{2}-2.79 X$

Valid for $\alpha_{c}=75^{\circ}$

$Z=5.82 X^{5}-21.40 X^{4}+25.89 X^{3}-8.33 X^{2}-1.98 X$

Valid for $\alpha_{c}=120^{\circ}$

$Z=-12.72 Y^{4}+25.44 Y^{3}-11.9 Y^{2}-0.82 Y$

Valid for the averaged non-dimensional transversal profile.

In the present study, the analysis was conducted in two steps. In the first step the effect of $\beta$ was analysed, fixing both the vertical angle and the crossing angle. It was proved that a non 
dimensional profile similitude can be pointed out, meaning that no significant effect on non dimensional scour hole profiles is due to $\beta$.

Figure 6a-b illustrates the non dimensional longitudinal $Z(X)$ and transversal $Z(Y)$ scour hole profile similitude occurring for $\alpha_{v}=60^{\circ}$ and $\alpha_{c}=30^{\circ}$ (along with Eq. 3) and for $\alpha_{v}=60^{\circ}$ and $\alpha_{c}=75^{\circ}$ (along with Eq. 6), respectively. In the second step, the effect of $\alpha_{v}$ on non dimensional scour hole profiles was taken into consideration and analysed. In this case, a profile similitude still occurs, even if a certain effect of vertical angle can be detected, as shown in Figure 7a-b, in which two examples of non dimensional longitudinal profile (for all tested $\alpha_{v}$ and $\alpha_{c}=30^{\circ}$ ) and transversal profile (for all tested $\alpha_{v}$ and $\alpha_{c}=75^{\circ}$ ) are reported, respectively. It can be observed that, for practical purposes, Eqs. (3)-(6) proposed by Pagliara et al. (2011a) can be reasonably generalized also for the case in which $\beta>0$. In addition, Pagliara et al. (2011a) observed that the maximum scour hole depth occurs for non dimensional longitudinal abscissa $X$ varying approximately between 0.5 and 0.65 . As the maximum scour hole generally takes place downstream of the jet crossing point, it implies that for scour design purposes, one can consider that the maximum backward extension (from the initial impact) should be surely less than $0.65 L_{c A}$ from the crossing point and for all the tested conditions.
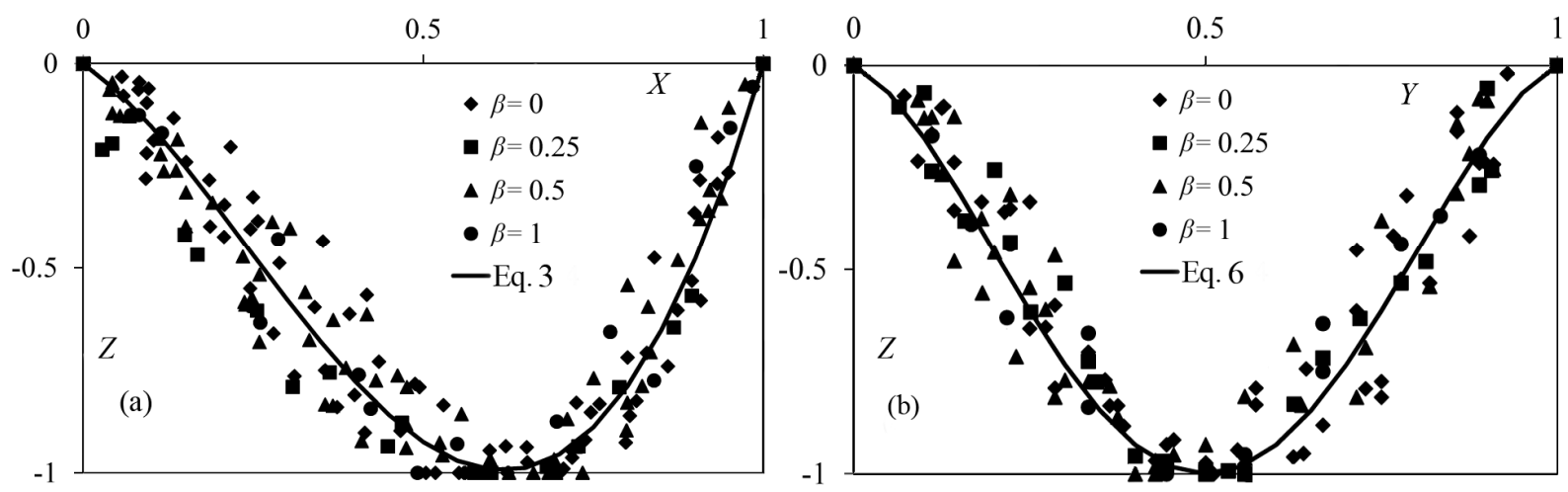

Figure 6(a) $Z(X)$ for $\alpha_{v}=60^{\circ}, \alpha_{c}=30^{\circ}$ and all the tested $\beta$ values along with Eq. (3); (b) $Z(Y)$ for $\alpha_{v}=60^{\circ}$, $\alpha_{c}=75$ and all the tested $\beta$ values along with Eq. (6)
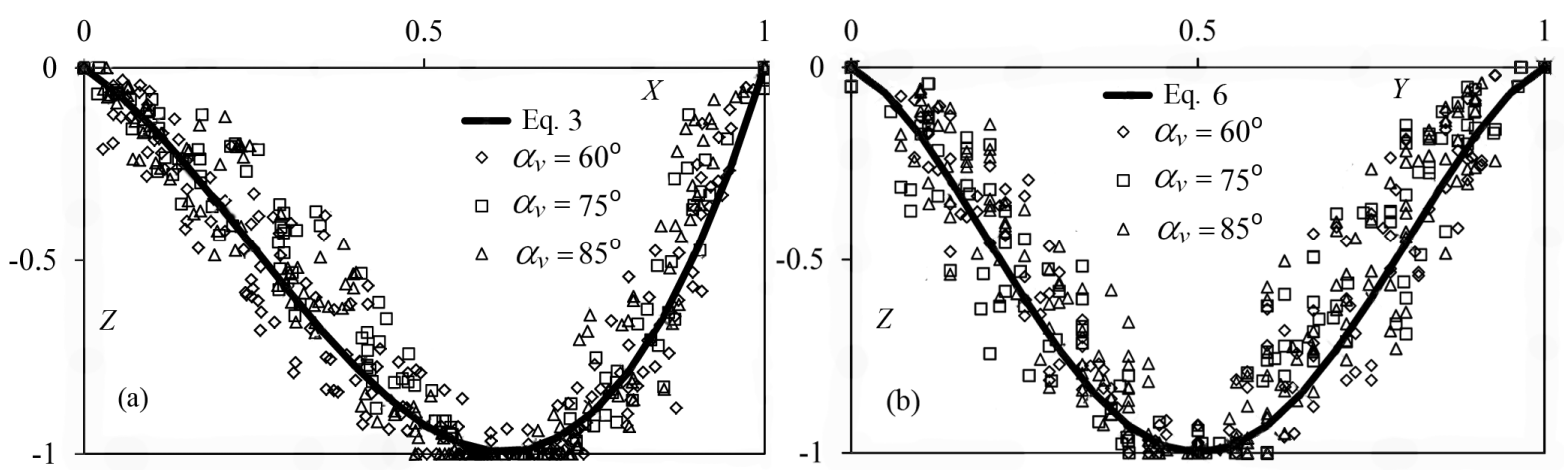
Figure 7 (a) $Z(X)$ for $\alpha_{c}=30^{\circ}$ and all the tested $\beta$ and $\alpha_{v}$ values along with Eq. (3); (b) $Z(Y)$ for $\alpha_{c}=75$ and all the tested $\beta$ and $\alpha_{v}$ values along with Eq. (6)

The analysis of non dimensional profiles was also conducted for the dune. In particular, both the longitudinal section of the dune A-A (see Figure 1a-b) and the transversal section C-C (i.e. the transversal section in which maximum dune height occurs) were analysed. According to the reference system adopted in the Figure 1, the following non dimensional coordinates can be defined: $X_{1}=\left(x-l_{c A}\right) / l_{M c A}$ and $Y_{1}=\left(y+b_{M c A} / 2\right) / b_{M c A}$, which are the non dimensional longitudinal and transversal coordinates of the dune in the mentioned sections, respectively. For all the tested conditions both the non dimensional longitudinal and transversal profiles were plotted as $Z\left(X_{1}\right)$ and $Z\left(Y_{1}\right)$, respectively. Also in this case, the effect of $\beta$ on $Z\left(X_{1}\right)$ was found negligible for all the hydraulic and geometric tested conditions. Moreover, the $Z\left(X_{1}\right)$ non dimensional profiles have a similar shape for each tested vertical angle $\alpha_{v}$. This occurrence allowed to find three different equations by which it is possible to predict the non dimensional longitudinal dune profiles depending only on $\alpha_{c}$ in the tested range of parameters. Equation (7) reports the analytical expression of the non dimensional longitudinal dune profiles. The coefficients $A, B, C$ and $D$ are listed in Table 3.

Figure 8a shows the average non dimensional longitudinal dune profiles for each $\alpha_{c}$ tested. It can be easily observed that increasing $\alpha_{c}$, the non dimensional profiles shift towards higher $X_{1}$ values. It means that increasing $\alpha_{c}$, the longitudinal shape of the dune becomes more symmetric. Namely, the non dimensional longitudinal coordinate in which the maximum dune height takes place approaches to 0.5 .
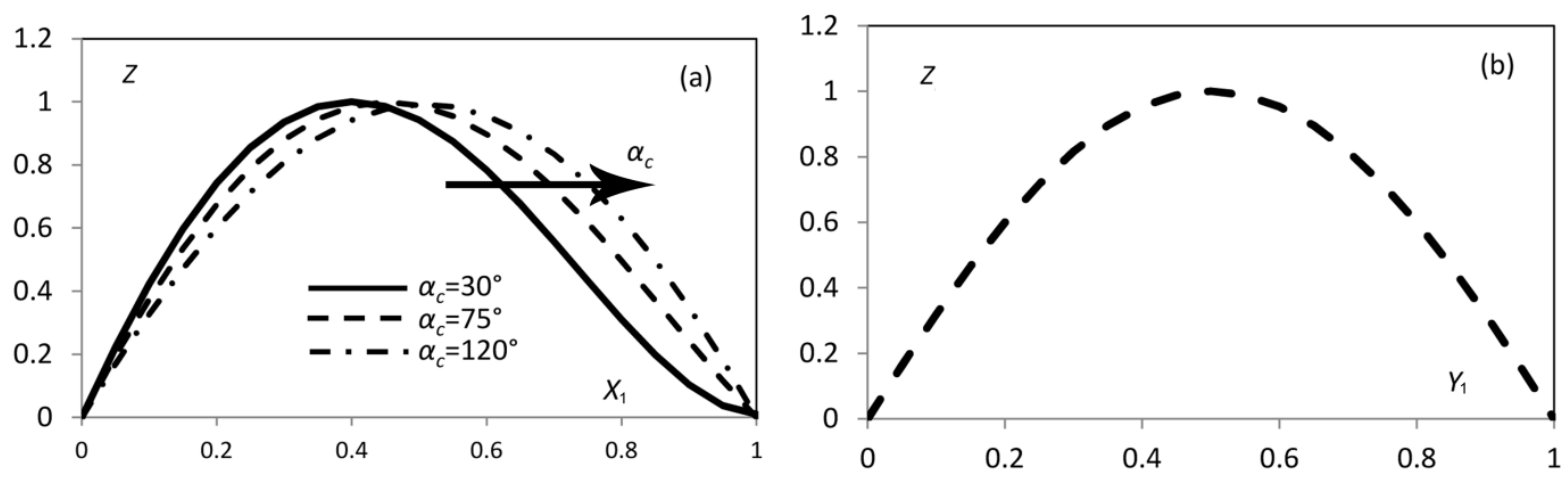

Figure 8(a) Average non dimensional longitudinal dune profiles $Z\left(X_{1}\right)$ : Eq. (7) for $\alpha_{c}=30^{\circ}, \alpha_{c}=75^{\circ}$, $\alpha_{c}=120^{\circ}$. (b) Average non dimensional transversal dune profiles $Z\left(Y_{1}\right)$ : Eq. (8) for all the tested conditions

The same analysis was conducted also for non dimensional transversal dune profiles (section 
$\mathrm{C}-\mathrm{C}$ in Figure 1b). In this case, it was experimentally proven that the non dimensional profiles $Z\left(Y_{1}\right)$ does not depend on both dimensionless flow rate $\beta$ and on the crossing angle $\alpha_{c}$. Thus a unique average transversal profile can be used to fit all the experimental data (Eq. 8), as shown in Figure 8b.

$Z=A X_{1}^{4}+B X_{1}^{3}+C X_{1}^{2}+D X_{1}$

valid for non dimensional longitudinal profiles

$Z=A Y_{1}^{4}+B Y_{1}^{3}+C Y_{1}^{2}+D Y_{1}$

valid for non dimensional transverse profiles

Table 3 Coefficients of Eqs (7) and (8)

\begin{tabular}{|c|c|c|c|c|}
\hline & \multicolumn{3}{|c|}{ Longitudinal } & Transverse \\
\hline$\alpha_{c}$ & $30^{\circ}$ & $75^{\circ}$ & $120^{\circ}$ & $30^{\circ}, 75^{\circ}, 120^{\circ}$ \\
\hline$A$ & 5.48 & 3.32 & 1.34 & 2.8 \\
\hline$B$ & -6.4 & -4.79 & -2.98 & -5.6 \\
\hline$C$ & -3.74 & -2.58 & -1.84 & -0.5 \\
\hline$D$ & 4.67 & 4.05 & 3.48 & 3.3 \\
\hline
\end{tabular}

\subsection{Scale effects}

In the present paper a detailed scale effects analysis was not conducted, also because of the complexity of the geometric configuration of the experimental apparatus. However, few observations can be done. In the presence of two jets crossing each other, it is not at all obvious and not even easily possible to detect which is the eventual influence on the scour hole mechanism of the scale effects characterizing the single jets. This is also due to the fact that the physical modeling of the scour phenomenon, based on Froude similitude, implies that the Weber number differs between experiments and that surface tension-dominated processes might not be properly scaled. However, the experiments performed in the present study are in a range of investigation which is close to that investigated by Chanson et al (2004) in Model 1. They analyzed the scale effects affecting air entrainment and bubble dispersion putting in evidence significant scale effects in terms of void fraction and bubble count rate when $\mathrm{We}<1000$, where $\mathrm{We}$ is the inflow weber number. Nevertheless, they observed a faster detrainment rate for $\mathrm{We}>1000$. This implies that a more specific analysis of the scale effects has to be conducted testing different models. Specific measurements are required to deep these aspects and they can be further developed in future investigations. 


\section{CONCLUSIONS}

The analysis of scour features due to crossing jets was conducted. This type of configuration can be of interest for practical applications as, especially in arch dams, it can occur and generally it can lead to a reduction of the erosive capacity of the resulting jet beyond the crossing point. Both the geometric configurations of the jets and hydraulic conditions were varied. Namely, three different crossing and vertical angles were tested; the discharge and the tailwater level were varied too. The analysis aimed to understand the effect of air content in the jets, thus experiments were carried out varying the ratio between air and water discharge. Moreover, reference tests (in absence of air) were also conducted in order to compare the scour morphology in the same hydraulic conditions and configuration, but with different dimensionless flow rates. It was experimentally proven that increasing dimensionless flow rate, the scour depth substantially reduces. An empirical relationship was proposed in order to determine the scour length parameters when effect of air plays a considerable role in scour process. The proposed relationship is a generalization of the equation proposed by the same authors for black water conditions. Finally, both the scour hole and dune morphologies were analysed. Similitudes between non dimensional profiles were pointed out and it was proved that, for practical purposes, the equations proposed for black water conditions can satisfactorily predict the analysed non dimensional profiles also in the case of aerated jets.

\section{REFERENCES}

Canepa, S. and Hager, W.H. (2003), Effect of jet air content on plunge pool scour, Journal of Hydraulic Engineering 129 (5), 358-365.

Chanson, H., Aoki, S., and Hoque, A. (2004), Physical modeling and similitude of air bubble entrainment at vertical circular plunging jets, Chemical Engineering Science 59 (4), 747-754.

Ervine, D.A. and Falvey, H.T. (1987), Behaviour of turbulent water jets in the atmosphere and in plunge pools in Proceedings of the Institute of Civil Engineers, part 2-Research and Theory, Vol. 83, pp. 295-314.

Hoffmans, G.J.C.M. (1998), Jet scour in equilibrium phase, Journal of Hydraul. Engineering 124 (4), 430-437.

Li, L., Liao, H. and Li, T. (2006), A hybrid model for simulating velocity field of a river with complex geometry plunged by multiple jets, Journal of Hydrodyn. 18 (6), 752-759.

Manso, P.A., Bollaert E.F.R. and Schleiss, A.J. (2007), Impact of turbulent high-velocity jets 
plunging in pools with flat bottom, Experiments in Fluids, 42(1), 49-60.

Mih, W.C. (1982), Scouring effects of water jets impinging on non-uniform streambeds, in Smith, P.E., “Applying Research to Hydraulic Practice”, ASCE, New York, pp. 270-279.

Mih, W.C. and Kabir, J. (1983), Impingement of Water Jets on Non uniform Streambed, Journal of Hydraulic Engineering 109 (4), 536-548.

Pagliara, S., Hager, W.H. and Minor, H. E. (2006), Hydraulics of plane plunge pool scour, Journal of Hydraulic Engineering 132 (5), 450-461.

Pagliara, S., Palermo, M. (2008), Plane plunge pool scour with protection structures, Journal of Hydro-Environment Research2(3), 182-191.

Pagliara, S., Amidei, M. and Hager, W.H. (2008), Hydraulics of 3D plunge pool scour, Journal of Hydraulic Engineering 134 (9), 1275-1284.

Pagliara, S., Roy, D. and Palermo, M. (2009), Effect of jet air content on 3D plunge pool scour, in IAHR Proceedings of 33rd IAHR Congress Water Engineering for a Sustainable Environment, Vancouver, August 9-14, 2009, pp. 3588-3595.

Pagliara, S., Roy, D., Palermo, M. (2010), 3D plunge pool scour with protection measures, Journal of Hydro-Environment Research 4(3), 225-233.

Pagliara, S., Palermo, M. and Carnacina, I. (2011a), Scour process due to symmetric dam spillways crossing jet, International Journal of River Basin Management 9(1), 31-42.

Pagliara, S., Palermo, M. and Roy, D. (2011b), Analysis of scour characteristics in presence of aerated crossing jets, in IAHR Proceedings of 34th IAHR World Congress - Balance and Uncertainty, Brisbane, Australia, 26 June - 1 July, pp. 2538-2545.

Rajaratnam, N. and Berry, B. (1977), Erosion by Circular Turbulent Wall Jets, Journal of Hydraulic Research 15(3), 277-289.

Rajaratnam, N. and Macdougall, R.K. (1983), Erosion by Plane Wall Jets with Minimum Tailwater, Journal of Hydraulic Engineering 109(7), 1061-1064. 